\title{
Simulation Research in Mutualism Behavior of Automobile Industry Cluster
}

\author{
Chen Zhang \\ Business College, University of Shanghai for Science and Technology \\ Room 601, No.11, 1111 Lane, Liang Cheng Road, Shanghai 200434, China \\ Tel: +86-139-177-87936 E-mail:zhangchen0505@126.com \\ Guangle Yan (Corresponding author) \\ Business College, University of Shanghai for Science and Technology \\ P.O. box No.70, Business College, Shanghai 200090, China \\ Tel: +86-021-6571-1857_E-mail:glyan2003@yahoo.com.cn
}

Received: January 18, 2011 Accepted: February 21, $2011 \quad$ doi:10.5430/jms.v2n1p41

The research is supported by the Innovation Fund Project for Graduate Student of Shanghai. No.JWCXSL1002, and the Shanghai Leading Academic Discipline Project. No. S30504.

\begin{abstract}
This paper adopts the concept and mechanism of mathematical ecology theory, researches the revolutionary of automobile industry chain by setting up mutualism model of two species. Results of simulation show that automakers are more sensitive to the change of environmental resource. Automakers depending on suppliers excessively will increase the structural risk of the industry. Suppliers must make efficient utilization of the system resources; otherwise they inhibit the growth of automakers.
\end{abstract}

Keywords: Automobile industry cluster, Simulation, Mutualism, Evolution

\section{Introduction}

With the growth of industry economy, industrial agglomeration is becoming main form of industry. Porter (1998) defined industry clusters as geographic concentrations of interconnected companies and institutions in a particular field. Industry clusters could effectively combine the advantage of industry development and the regional economy, resolve conflicts between specialization and transaction convenience, thus forming efficient production organization way. Enterprises compose industry cluster, nonetheless industry is not simply summation of enterprises. Interaction of enterprises makes them to form an organic unity.

Population is all the organisms that both belong to the same species and live in the same geographical area. Population has the following characters: exchange energy with external environment during development, and internal ecosystem could regulate itself to evolution. There is comparability between ecological relationship and mechanism of evolution of industry clusters and population. Therefore more and more study is applied from the perspective of ecology. Jianxuan Chen (2004) proposed to use ecology theory to research industry clusters. In the study on evolution stability of industry clusters, Jishan He (2005) set up competition and benefit model of upstream and downstream relationship. Baoxing Qiu(1999) proposed that each enterprises should find its eco-logical niche to remain cluster stable. Faming Wang (2009) took bicycle industry cluster in Taiwan as example, studied the formation and evolutionary path of industrial clusters. Jin Zhao (2010) judged the ecological stability of industry clusters by using loop analysis. In study of relationship in the group, Jian Li (2006), Hao Zhou (2003) respectively studied the ecological characters of clusters and symbiosis models of different structural clusters by setting up a logistic model. Pingnan Ruan (2008) established two species model to research positive effect from competition and synergetic to the evolution of industry. However, these study are mainly qualitative analysis, the interpretation of evolution mechanisms is not elaborate. In this paper, the two species model is introduced to set up a mutualism model of Automobile Industry Chain, then explanation of mutualism behavior of vehicle manufacturers and vehicle parts suppliers. 


\section{Model Summery}

Assume a biological community consists of $n$ species groups, and $n \geq 2$. When $n=2$, there are two species groups in the biological community, they interact with each other and compose the simplest biological community. There are two types of relationship between the species: the neutral one means one species has no affection to the other one; the other one means two species has affection to each, the affection is usually divided into three kinds: competition, mutualism and prey. In this paper, relationship of affection to each other is been considered.

\subsection{Lotka-Volterra Two Species Model}

A.j.Lotka and V.Volterra set up predator-prey model and competition model separately. The models above were extended to mutualism system by Odum. The later researchers called the three models as Lotka-Volterra model. It is:

$$
\left\{\begin{array}{l}
d x_{1} / d t=x_{1}\left(b_{1}+a_{11} x_{1}+a_{12} x_{2}\right) \\
d x_{2} / d t=x_{2}\left(b_{2}+a_{21} x_{1}+a_{22} x_{2}\right)
\end{array}\right.
$$

Assumptions of this model are:

(1) $a_{11}<0$ and $a_{22}<0$, means the two species are both restricted by density of themselves. (2)If $a_{12}=0, a_{21}>0$ ( or $a_{12}>0, a_{21}=0$ ), means $x_{1}$ is of benefit to $x_{2}$ (or $x_{2}$ is of benefit to $x_{1}$ ). (3)If $a_{12}=0, a_{21}<0$ (or $\left.a_{12}<0, a_{21}=0\right)$, means $x_{1}$ is harmful to $x_{2}$ (or $x_{2}$ is harmful to $\left.x_{1}\right)$. (4) $b_{2}<0$ means natural mortality rate of heterotrophy such as predators. (5)If $b_{1}<0$ and $b_{2}<0$, mortality rate of the two species, and neither of them could survive in the other's absence. (6)If $b_{1}>0$ and $b_{2}<0$, means species $x_{1}$ can survive without $x_{2}$, but $x_{2}$ cannot without $x_{1}$. (7)Signs of coefficient $a_{i j}(i=1,2 ; j=1,2)$ decide which kind of system it is. If $a_{12}<0, a_{21}>0$, formula (1) is a predator-prey system; while $a_{12}<0, a_{21}<0$, formula (1) is a competition system; when $a_{12}>o, a_{21}>0$, formula (1) is a mutualism system.

\section{Mutualism Model of Industry Clusters}

With manufacture as its flagship, industrial clusters boast full-fledged markets in professional components and parts manufacturers, machinery equipment and related service suppliers and associated industries. Therefore, the enterprises in industrial clusters can be classified as manufacturing enterprises and spare part enterprises-the downstream industrial chain, both of which are mutually dependent and beneficial. In ecology, this mutually beneficial relationship is categorized as two kinds: one is mutualistic symbiosis, also called obligate mutualistic symbiosis: either part will die if it is parted from the other. The other is proto-cooperation, also called facultative mutualistic symbiosis: cooperation between the two symbiotic parts is loose and both parts can exist independently when they are parted from each other, like the relationship between bees and various plants. For automobile industrial clusters, entire automobile manufacturers and spare part suppliers can enjoy a lower cost brought by contiguous locations, winning a price advantage over industries outside the clusters. Therefore, we hold the view that the relationship between the two enterprises within the industrial cluster is mutualistic symbiosis, that is, one can not exist when it is parted from the other.

\subsection{Mutualism System Model}

Suppose enterprises within the industrial cluster perish exponentially without partnership, the simplest model of mutualistic symbiosis Lotka-Volterra can be deduced as follows:

$$
\left\{\begin{array}{l}
d x_{1} / d t=-c_{1} x_{1}+P_{1} x_{1} x_{2} \\
d x_{2} / d t=-c_{2} x_{2}+P_{2} x_{1} x_{2}
\end{array}\right.
$$

$\mathrm{P}>01$ and $\mathrm{P} 2>0$, which signifies the mutual benefit between these two kinds of enterprises.

\subsection{Improvement of Mutualism System Model}

In the real-life predator-prey system, the number of various predators increases in different degree, while the total resource of the system is not infinite. Therefore, it is impossible for both predator and prey to proliferate without limitation. Based on this, we have to improve the mutualistic symbiosis model by adding constraint conditions so as to make it approximate the reality. On the basis of experiments and research, Holling provided three different nutrition functions $\varphi(x)$ for predators of different types. 


$$
\mathrm{I}: \varphi(x)=\left\{\begin{array}{l}
b x / a, x \leq x \leq a \\
b, x>a
\end{array}\right.
$$

Which is also called linear response, describing the situation when the number of predators become saturated if the food supply is plentiful.

$$
\text { II : } \varphi(x)=a x /(1+a x)
$$

Describing the situation that the consumption of predators increases up to saturation with the increase of the prey. When the food supply is sufficient, starving degree of predators decreases.

$$
\text { III: } \varphi(x)=a x^{2} /\left(1+b_{1} x^{2}\right)
$$

when the food supply is insufficient, formula (5) describing the uniquely complicated behaviors of vertebrates which increase their consumption by learning preying skills, When the food amount outnumbers a certain amount $x_{0}$, the starving degree of predators decreases and predators' consumption increases negatively till when the number of predators becomes saturated. Herein, suppose the nutrition function of automobile industry be the second one.

\section{Simulation Study}

In automobile industrial cluster, the entire automobile manufacturers are predators who can make maximal use of the resources provided by the spare part suppliers to realize the optimal development of their own when the spare part supply is sufficient. When the scale and number of entire automobile manufacturers exceed certain extent, land and capital within the industrial cluster are not enough for the spare part manufacturers to keep pace with them. At this moment, the entire automobile manufacturers within the industrial cluster will encounter a bottleneck which may slow down or stop their development. Define $x_{1}$ as the entire automobile manufacturers which are the predators in the community and $x_{2}$ as the spare part suppliers which are the prey in the community. The following model is set as:

$$
\left\{\begin{array}{l}
d x_{1} / d t=-c_{1} x_{1}+P_{1} x_{1} x_{2} /\left(1+D_{1} x_{2}\right)-e_{1} x_{1}^{2} \\
d x_{2} / d t=-c_{2} x_{2}+P_{2} x_{1} x_{2} /\left(1+D_{2} x_{1}\right)-e_{2} x_{2}^{2}
\end{array}\right.
$$

$c_{1}>0$ and $c_{2}>0$ represent their declining rate respectively when there is a lack of symbiosis parnters. $P_{i} x_{1} x_{2}(i=1,2)$ represents the symbiosis effect of entire automobile manufacturers and spare part suppliers. $P_{1}>0$ indicates the increasing rate of entire automobile manufacturers relying upon spare part suppliers and $P_{2}>0$ the the increasing rate of spare part suppliers under the mutualistic symbiosis effect. $\left(1+D_{i} x_{j}^{2}\right) D_{i}>0 i=1,2 ; j=2,1$ are the divisors of nutrition function describing the increasing path of entire automobile manufacturers when the spare part suppliers are limited, which appears as the reciprocal of resource utilization rate of entire automobile manufacturers to spare part suppliers; vice versa, the reciprocal of resource utilization rate of the spare part suppliers under the support of the entire automobile manufacturers. The more $D_{i}$ is, the less is the resource utilization rate of the mutualistic symbiosis partner. $e_{i}>0$ represents the constraint coefficient of land and capital resources within the whole industrial cluster.

\section{Results and Analysis}

If there is no special explanation, the emulated parameter values are as follows: $t=(0,10)$, $x_{1}=585,(t=0), x_{2}=198,(t=0), c_{1}=0.5, c_{2}=0.3, P_{1}=1.2, P_{2}=0.75, D_{1}=0.8, D_{2}=0.9, e_{1}=0.003$, $e_{2}=0.001$.

In order to understand the influence of different parameters upon the system stability, the parameters are further revised for the sake of the system's convergence and stability.

\subsection{Influence of initial value upon the system}

Change initial value of parameters to test the system. Let $x_{1}=300,(t=0), x_{1}=200,(t=0), x_{2}=300,(t=0)$; $x_{1}=200,(t=0), x_{2}=600,(t=0)$. Table 1 is a simulation gained by adjusting the initial values of the system. From table 1, we can see that no matter what the initial values are, $x_{1}, x_{2}$ will be converged to achieve a stable level with $x_{1}<x_{2}$. It indicates that when the system is stable, the number of suppliers is more than manufacturers in the relationship of mutualistic symbiosis so as to satisfy the need of the latter. 


\subsection{Influence of declining rate upon the system}

Simulation results are gained by giving values to the declining rate of entire automobile manufacturers and spare part suppliers respectively when there are no symbiosis partners in the analytical system. Let $c_{1}=0.3, c_{2}=0.5, c_{1}=0.03$, $c_{2}=0.05, c_{1}=0.4, c_{2}=0.12$. Table 2 shows the results. The declining rate determines who has a comparatively advantageous number in the industrial cluster after the system becomes stable. When the declining rates are in proportion, that is $c_{1} / c_{2}=C$, there is hardly change of the system which will be prone to stability after a certain period.

5.3 Influence of mutualism effect upon the system

Parameter $P_{i}$ is adjusted and given value as $P_{1}=0.75, P_{2}=0.3 ; P_{1}=0.75, P_{2}=1.2 ; P_{1}=1.2, P_{2}=0.5$. From the simulation result in table 3 , we can conclude that when the symbiosis effect parameter of the entire automobile manufacturers becomes stable, with the speedy increase of that of the spare part suppliers, the value of entire automobile manufacturers dives sharply and it takes a longer time to become stable again. It reveals that the excessive development of satellite industry such as spare part suppliers within a industrial cluster will overuse the capital and land needed by the leading industries, which can not help the leading industries' development but slow down their speed. From the simulation result obtained from the third group of parameters, when the symbiosis effect parameter of the entire automobile manufacturers is too big, the amount of entire automobile manufacturers increases negatively under the significant influence of symbiosis effect. This reason for the low productive efficiency is that the entire automobile manufacturers depend too much upon the spare part suppliers without making good use of other resources within the system.

\subsection{Influence of divisor of the nutrition function upon the system}

The simulation result is showed in table 4 by analysing the resource utilization rate of entire automobile manufacturers to spare part suppliers. When the divisor of the nutrition function of entire automobile manufacturers is bigger than that of spare part suppliers, that is, when the resource utilization rate of entire automobile to spare part is higher than the vice versa, both parts within the cluster will increase up to a stable level. Vice versa, the increase of the entire automobile manufacturers will be restricted and even decline with the increase of spare part suppliers. After a certain period, it will be prone to stability again. The reason for this phenomenon is that the limited resources are over-consumed due to the rapid increase of the spare part suppliers. When the resource utilization rate of either part to the other is low, the productivity of entire automobile manufacturers will drop substantially and will not become stable until it approximates the level of spare part suppliers.

\subsection{Influence of environmental resource restrictions upon the system}

Let $e_{1}=0.002, e_{2}=0.007 ; e_{1}=0.007, e_{2}=0.007, e_{1}=0.03, e_{2}=0.01$, table 5 shows the More restrictions to spare part suppliers will affect the entire automobile manufacturers to a certain extent. When there are more restrictions to the entire automobile and invariant restrictions to the spare part, the productivity of the entire automobile will fall sharply but that of the spare part will hardly drop. It indicates that the structural relationship between the two parts will not be influenced by the environmental resources. When both parts suffer equal restrictions by environmental resources, the productivity of entire automobile manufacturers drops more magnificently than spare part suppliers and then achieves stability on a lower level. It shows that creatures on the top of food chain are more vulnerable to the environmental resources.

\section{Conclusions}

In the preliminary stage of automobile industrial cluster, the entire automobile manufacturers are the leading enterprises while the spare part manufacturers and their related lower-stream industries are under-developed, which can be demonstrated as $P_{2}>P_{1}>0$, that is, the leading industries contribute more to the satellite industries. When the automobile industry becomes increasingly mature with balanced development between leading and lower-stream industries, represented as $P_{1} \approx P_{2}$, the intensity of enterprise distribution and cooperation between the two kinds of enterprises exert a significant influence on the growth of enterprises within the industrial cluster. On the top of the food chain, the entire automobile manufacturers are more sensitive to the environmental resources. Besides, their over-dependence upon spare part suppliers will raise structural risk of the industrial cluster. If the spare part suppliers are not able to make sufficient use of resources within the cluster during their speedy development, they may bring restraints upon the entire automobile manufacturers.

Hereby, we suggest that enterprises within the industrial cluster should readjust their industrial structures and increase the resource utilization rate between entire automobile manufacturers and spare part suppliers. In addition, enterprises should strengthen their innovation ability so as to take an flexible and active position among the symbiosis objects in the system. Meanwhile, the system should be in open and flowing situation with well-integrated resources rather than blind expansion of production. 


\section{References}

Porter. M. (1998). Clusters and New Economies of Competition. Harvard Business Review, 76, pp. 77-90

Jianxuan Chen, \& Jianmei Yang, (2004). Study on enterprises cluster based on ecosystem. Technoeconomics \& Management Research, 5, pp. 22-23

Jishan He, \&Weiming Dai, (2005). The equilibrium of industrial clusters: from an ecological perspective. Journal of Beijing Normal University. Natural Science, 1, pp. 126-132

Baoxing Qiu, (1999). Study on small enterprises cluster. Shanghai: Fudan University Press, (Chapter 3).

Faming Wang, (2009). On industrial population and industrial cluster and their evolution. Productivity Research, 5, pp. $124-127$

Jin Zhao, \&Yanping Liu, (2010). The Co-evolution of industrial cluster ecosystem based on loop analysis, Scientific management Research, 28, pp. 70-72

Jian Li, \&Zhanming Jin, (2006). The development of industrial cluster based on the zoology theory. Studies in Science of Science, 24, pp. 431-437

Hao Zhou, (2003). Enterprises cluster co-existence model and stability analysis. Systems Engineering, 24, pp. 32-37

Pingnan Ruan, \&Lei Huang, (2008). Study on evolutionary dynamics of industrial clusters: from an ecological perspective. Enterprise Economy, no.6, pp. 5-8

Table 1.Influence of initial value upon the system

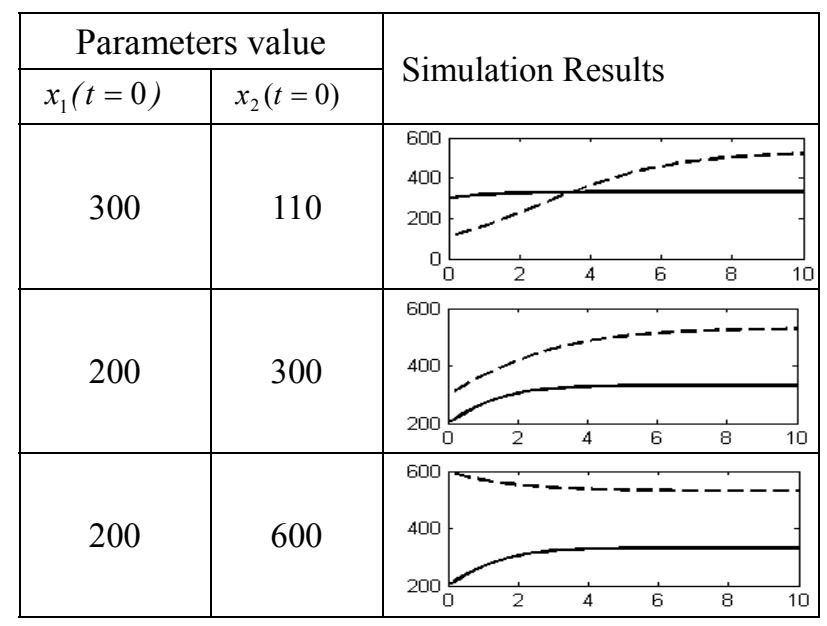

Table 2. Influence of declining rate upon the system

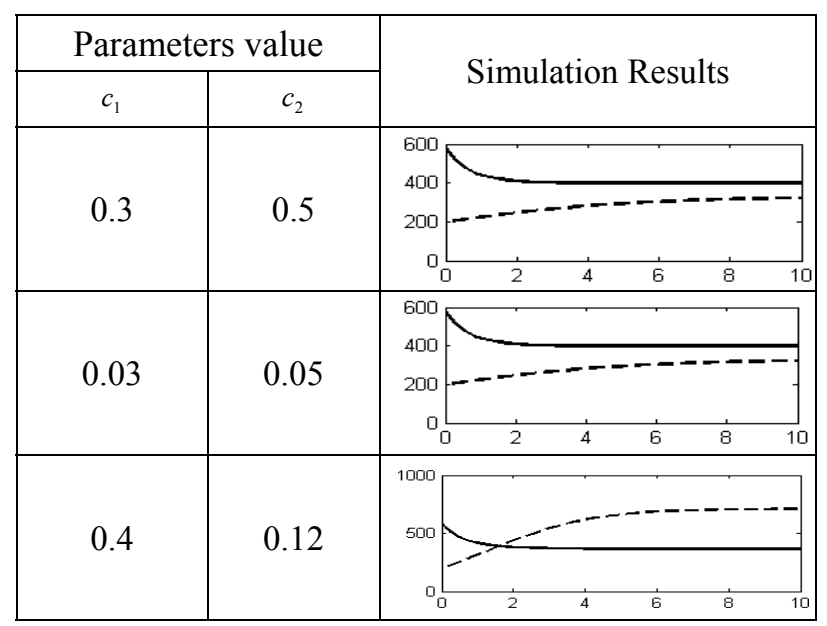


Table 3. Influence of mutualism effect upon the system

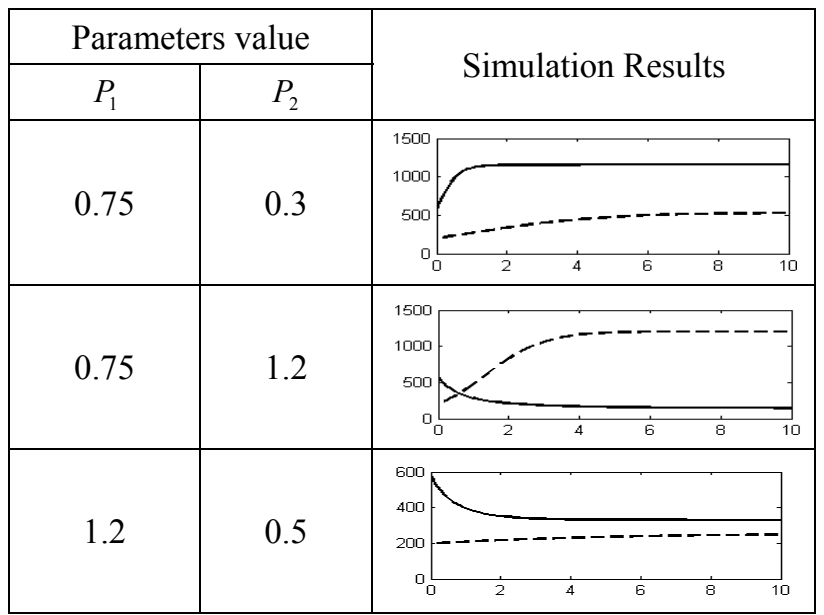

Table 4. Influence of divisor of the nutrition function upon the system

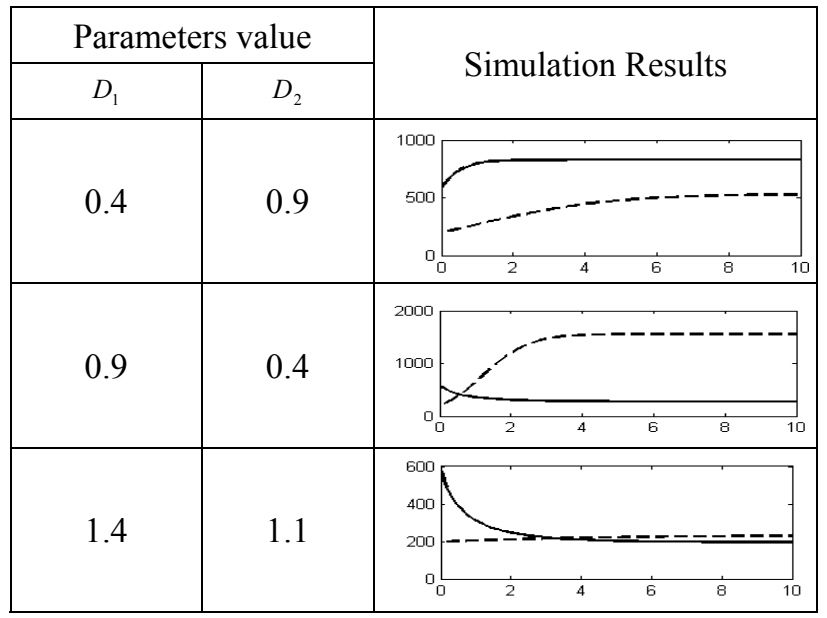

Table 5. Influence of environmental resource restrictions upon the system

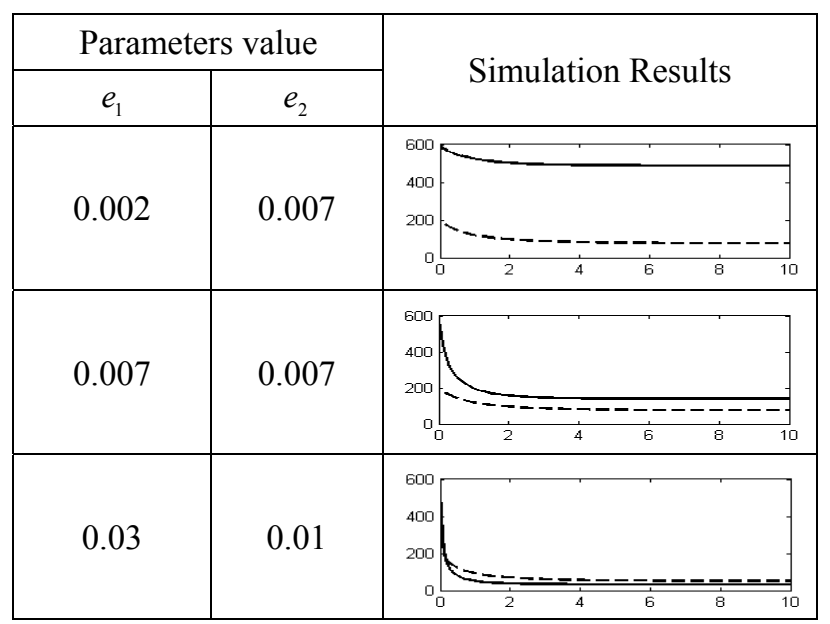




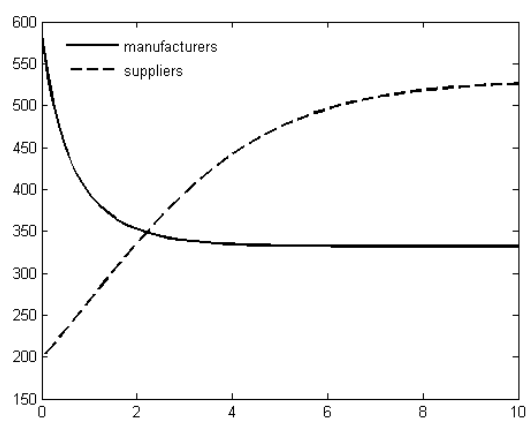

Figure 1.Simulation Result 